\title{
Exploring the link between project management approach and project success dimensions: A structural model approach
}

\author{
Ciric, D. ${ }^{a}$, Delic, M. ${ }^{\mathrm{a},{ }^{*}}$, Lalic, B. ${ }^{a}$, Gracanin, D. ${ }^{\mathrm{a}}$, Lolic, T. ${ }^{\mathrm{a}}$ \\ a University of Novi Sad, Faculty of Technical Sciences, Novi Sad, Serbia
}

\begin{abstract}
A B S T R A C T
Aligning the project management approach to a particular project is considered to be essential for project success. Based on the literature review, in this research, the project management approach is analyzed through differentiation between agile and traditional approaches within the specific managerial aspects. This research aimed to contrast these two project management approaches and explore their impact on different project success dimensions. The research was conducted on a sample of 227 project management professionals worldwide, using the PLS-SEM method. Research results denounced that, in most cases, the traditional approach is applied in project initiation and planning. It has proven to provide higher-level od project success, while, within all other managerial aspects considered in this research, they gravitate more towards the agile approach. By combining agile and traditional approaches, organizations can take advantage of some benefits of agile development without abandoning the stability provided by a traditional approach. The study is relevant for project management practitioners tailoring down the success-oriented project management approach and developing project management contingency theory for academics.
\end{abstract}

\begin{tabular}{l} 
A R T I C L E I N F O \\
\hline Keywords: \\
Project management approach; \\
Agile; \\
Traditional; \\
Project success; \\
Structural-model approach \\
*Corresponding author: \\
delic@uns.ac.rs \\
(Delic, M.) \\
Article history: \\
Received 31 January 2020 \\
Revised 9 February 2020 \\
Accepted 13 February 2020 \\
$\begin{array}{l}\text { Cc } \quad \text { B } \\
\text { BY }\end{array}$ \\
Content from this work may be used under the terms of \\
the Creative Commons Attribution 4.0 International \\
Licence (Cc BY 4.0). Any further distribution of this work \\
must maintain attribution to the author(s) and the title of \\
the work, journal citation and DoI.
\end{tabular}

\section{References}

[1] Gajic, S., Palcic, I. (2019). A new framework for complexity analysis in international development projects Results from a Delphi study, Advances in Production Engineering \& Management, Vol. 14, No. 2, 225-238, doi: 10.14743/apem2019.2.324.

[2] Wysocki, R.K. (2019). Effective project management: Traditional, agile, extreme, hybrid, Eighth edition, John Wiley \& Sons, Indianapolis, USA, doi: 10.1002/9781119562757.

[3] Sauser, B.J., Reilly, R.R., Shenhar, A.J. (2009). Why projects fail? How contingency theory can provide new insights - A comparative analysis of NASA's Mars climate orbiter loss, International Journal of Project Management, Vol. 27, No. 7, 665-679, doi: 10.1016/j.ijproman.2009.01.004.

[4] Sheffield, J., Lemétayer, J. (2013). Factors associated with the software development agility of successful projects, International Journal of Project Management, Vol. 31, No. 3, 459-472, doi: 10.1016/i.ijproman.2012. 09.011.

[5] Fernandez, D.J., Fernandez, J.D. (2008). Agile project management - Agilism versus traditional approaches, Journal of Computer Information Systems, Vol. 49, No. 2, 10-17, https://www.tandfonline.com/doi/abs/10.1080/ $\underline{08874417.2009 .11646044 .}$

[6] Janekova, J., Fabianova, J., Fabian, M. (2019). Assessment of economic efficiency and risk of the project using simulation, International Journal of Simulation Modelling, Vol. 18, No. 2, 242-253, doi: 10.2507/IJSIMM18(2)467.

[7] Berinato, S., The secret to software success, from https://www.cio.com/article/2441472/the-secret-to-softwaresuccess.html, accessed July 1, 2018.

[8] Larman, C. (2004). Agile and iterative development, A manager's guide, Addison-Wesley Professional, Boston, USA. 


\section{APEM}

\title{
Raziskovanje povezave med pristopom projektnega vodenja in razsežnostmi uspeha projekta: Pristop strukturnega modeliranja
}

\author{
Ciric, D. ${ }^{a}$, Delic, M. ${ }^{\text {a, }}{ }^{,}$, Lalic, B. ${ }^{a}$, Gracanin, D. ${ }^{\text {a }}$, Lolic, T. ${ }^{\text {a }}$ \\ aUniversity of Novi Sad, Faculty of Technical Sciences, Novi Sad, Serbia
}

\section{POVZETEK}

Izbira pristopa projektnega vodenja za določen projekt je bistvenega pomena za uspeh projekta. Na podlagi pregleda literature je $\mathrm{v}$ tej raziskavi pristop projektnega vodenja analiziran s primerjavo agilnega in tradicionalnega pristopa v okviru specifičnih upravljavskih vidikov. Namen te raziskave je bil primerjati ta dva pristopa projektnega vodenja in raziskati njun vpliv na različne razsežnosti uspeha projekta. Raziskava je bila izvedena na vzorcu 227 strokovnjakov s področja projektnega vodenja po vsem svetu po metodi PLS SEM. Rezultati raziskave so pokazali, da se v večini primerov pri zagonu in načrtovanju projekta uporablja tradicionalni pristop. Izkazalo se je, da tradicionalni pristop zagotavlja višjo stopnjo uspešnosti projekta. V okviru vseh ostalih upravljavskih vidikov, obravnavanih v tej raziskavi, pa je agilni pristop boljša izbira. S kombinacijo agilnega in tradicionalnega pristopa lahko organizacije izkoristijo nekatere prednosti agilnega vodenja, ne da bi opustile stabilnost, ki jo zagotavlja tradicionalni pristop. Študija je pomembna za izvajalce projektnega vodenja, ki uporabljajo k uspehu usmerjen pristop projektnega vodenja in razvijajo kontingenčno teorijo projektnega vodenja.
\end{abstract}

\section{PODATKI O ČLANKU}

Ključne besede:

Pristop projektnega vodenja

Agilnost;

Tradicionalnost;

Uspeh projekta;

Pristop strukturnega modeliranja

* Kontaktna oseba:

delic@uns.ac.rs

(Delic, M.)

\section{Zgodovina članka:}

Prejet 31. januarja 2020

Popravljen 9. februarja 2020

Sprejet 13. marca 2020

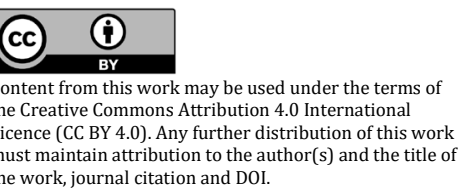


[9] Lindstrom, L., Jeffries, R. (2004). Extreme programming and agile software development methodologies, Information Systems Management, Vol. 21, No. 3, 41-52, doi: 10.1201/1078/44432.21.3.20040601/82476.7.

[10] Vinekar, V., Slinkman, C.W., Nerur, S. (2006). Can agile and traditional systems development approaches coexist? An ambidextrous view, Information Systems Management, Vol. 23, No. 3, 31-42, doi: 10.1201/1078.10580530/ 46108.23.3.20060601/93705.4.

[11] Hair, J.F., Hult, G.T.M., Ringle, C., Sarstedt, M. (2014). A Primer on Partial Least Squares Structural Equation Modeling (PLS-SEM), Sage, Thousand Oaks, USA.

[12] Tripathi, V., Goyal, A.K. (2014). Changing roles and responsibilities from traditional project management to agile project management, International Journal on Recent and Innovation Trends in Computing and Communication, Vol. 2, No. 5,1005-1009.

[13] Serrador, P., Pinto, J.K. (2015). Does agile work? - A quantitative analysis of agile project success, International Journal of Project Management, Vol. 33, No. 5, 1040-1051, doi: 10.1016/j.ijproman.2015.01.006.

[14] Project Management Institute. PMI's pulse of the profession: Capturing the value of project management through organizational agility, from http://www.pmi.org/ /media/PDF/learning/translations/2015/capture-valueorganizational-agility.ashx, accessed July 1, 2018.

[15] Castro-Ríos, G.A., Reyes-Rubiano, R.S., Noguera-Hidalgo, Á.L. (2017). What are management systems? The effect of management style according to system dynamics, International Journal of Industrial Engineering and Management, Vol. 8,No. 2, 61-69.

[16] Špundak, M. (2014). Mixed agile/traditional project management methodology - Reality or illusion?, Procedia Social and Behavioral Sciences, Vol. 119, 939-948, doi: 10.1016/i.sbspro.2014.03.105.

[17] Dybå, T., Dingsøyr, T. (2008). Empirical studies of software development: A systematic review, Information and Software Technology, Vol. 50, No. 9-10, 833-859, doi: 10.1016/i.infsof.2008.01.006.

[18] Imreh, R., Raisinghani, M. (2011). Impact of agile software development on quality within information technology organisations, Journal of Emerging Trends in Computing and Information Science, Vol. 2, No. 10, 460475.

[19] Ahimbisibwe, A., Cavana, R.Y., Daellenbach, U. (2015). A contingency fit model of critical success factors for software development projects: A comparison of agile and traditional plan-based methodologies, Journal of Enterprise Information Management, Vol. 28, No. 1, 7-33, doi: 10.1108/[EIM-08-2013-0060.

[20] Pinto, J.K., Slevin, D.P. (1988). Critical success factors across the project life cycle, Project Management Journal, Vol. 19, No. 3, 67-75.

[21] Shenhar, A.J., Dvir, D. (2007). Reinventing project management: The diamond approach to successful growth and innovation, Harvard Business School Press, Boston, USA.

[22] Shenhar, A.J., Levy, O., Dvir, D. (1997). Mapping the dimensions of project success, Project Management Journal, Vol. 28, No. 2, 5-13.

[23] Atkinson, R. (1999). Project management: Cost, time and quality, two best guesses and a phenomenon, its time to accept other success criteria, International Journal of Project Management, Vol. 17, No. 6, 337-342, doi: 10.1016/ S0263-7863(98)00069-6.

[24] Shenhar, A.J., Dvir, D., Levy, O., Maltz, A.C. (2001). Project success: A multidimensional strategic concept, Long Range Planning, Vol. 34, No. 6, 699-725, doi: 10.1016/S0024-6301(01)00097-8.

[25] Bourne, M., Mills, J., Wilcox, M., Neely, A., Platts, K. (2000). Designing, implementing and updating performance measurement systems, International Journal of Operations \& Production Management, Vol. 20, No. 7, 754-771, doi: $10.1108 / 01443570010330739$.

[26] Mir, F.A., Pinnington, A.H. (2014). Exploring the value of project management: Linking project management performance and project success, International Journal of Project Management, Vol. 32, No. 2, 202-217, doi: 10.1016/i.ipproman.2013.05.012.

[27] Hair, J.F., Black, W.C., Babin, B.J., Anderson, R.E. (2010). Multivariate data analysis, Seventh edition, Pearson Education, Upper Saddle River, New Jersey, USA.

[28] Nunnally, J.C., Bernstein, I.H. (1994). Psychometric Theory, Third edition,McGraw-Hill, New York, USA.

[29] Dillman, D.A., Smyth, J.D., Christian, L.M. (2008). Internet, mail, and mixed-mode surveys: The tailored design method, Third edition, John Wiley and Sons, Hoboken, New York, USA.

[30] Ciric, D., Lalic, B., Gracanin, D., Tasic, N., Delic, M., Medic, N. (2019). Agile vs. traditional approach in project management: Strategies, challenges and reasons to introduce agile, Procedia Manufacturing, Vol. 39, 1407-1414, doi: 10.1016/j.promfg.2020.01.314.

[31] Vulanovic, S., Delic, M., Kamberovic, B., Beker, I., Lalic, B. (2020). Integrated management systems based on risk assessment: Methodology development and case studies, Advances in Production Engineering \& Management, Vol. 15, No. 1, 93-106, doi: 10.14743/apem2020.1.352.

[32] Boehm, B., Turner, R. (2005). Management challenges to implementing agile processes in traditional development organizations, IEEE Software, Vol. 22, No. 5, 30-39, doi: 10.1109/MS.2005.129.

[33] Car-Pušić, D., Marović, I., Bulatović, G. (2020). Development of a hybrid agile management model in local selfgovernment units, Tehnički Vjesnik - Technical Gazette, Vol. 27, No. 5, 1418-1426, doi: 10.17559/TV-20190205 140719.

[34] Martinec, T., Škec, S., Savšek, T., Perišić, M.M. (2017). Work sampling for the production development: A case study of a supplier in European automotive industry, Advances in Production Engineering \& Management, Vol. 12, No. 4, 375-387, doi: 10.14743/apem2017.4.265.

[35] Wells, H. (2012). How effective are project management methodologies? An explorative evaluation of their benefits in practice, Project Management Journal, Vol. 43, No. 6, 43-59, doi: 10.1002/pmj.21302. 